$\mathrm{p}<0.05$ ) and 32\% (OR 0.68, 95\% CI: 0.53, 0.86, p < 0.05) decreased odds of future knee injuries, respectively. Results of best-evidence synthesis found that 10 of 15 proximal stability variables were not associated with future knee injuries.

Conclusions Hip extension and external rotation strength are the strongest predictors of future knee injuries. The majority of proximal stability variables included in this review were not associated with knee injuries. This review was limited by heterogeneity of measurement methods, making categorizing them difficult. Future studies should consider larger sample sizes to ensure adequate power, and the use of multivariable and complex systems approaches to account for the multifactorial nature of athletic injuries.

\section{CORE STABILITY AS A RISK FACTOR FOR THE DEVELOPMENT OF ACUTE LOWER EXTREMITY INJURIES IN AN ATHLETIC POPULATION: A PROSPECTIVE STUDY}

Cedric De Blaiser, Roel De Ridder, Tine Willems, Stefan Vermeulen, Lieven Danneels, Philip Roosen. Ghent University, Ghent, Belgium

\subsection{6/bjsports-2021-IOC.135}

Background Impaired core stability has been suggested to influence lower extremity functioning and might contribute to the development of acute lower extremity injuries. Prospective studies that examine this relationship are currently lacking.

Objective The objective of this study was to investigate the role of different components of core stability as risk factors for the development of acute lower extremity injuries.

Design A prospective study was set up with a follow-up and injury registration period of 1,5 years. Afterwards, cox regression analyses were performed to identify significant contributors in the development of acute lower extremity injuries.

Setting Male and female freshmen students, enrolled in the physical education teacher studies of the University Colleges in Ghent participated in this study. The study was performed at the Ghent Sports Arena.

Patients (or Participants) 150 healthy participants enrolled and were excluded if they had a history of lower extremity surgery or if they reported a musculoskeletal injury in the 6 months prior to testing. Eleven participants were excluded which resulted in 139 included participants.

Interventions (or Assessment of Risk Factors) Measures for dynamic postural control, isometric core and hip muscle strength, core muscle endurance, core neuromuscular control and proprioception were taken at baseline.

Main Outcome Measurements The occurrence of an acute lower extremity injury was the primary study outcome.

Results During follow-up, 27 injuries of interest occurred (19\%). After multivariate model building, a significant predictive effect was found for a muscle strength imbalance for hip flexion $(p=0.016)$. The risk of developing an injury increased with a greater strength imbalance, regardless of sex and other core stability measures.

Conclusions This study identified hip strength imbalance as a risk factor for the development of acute lower extremity injuries. Normalizing hip strength imbalance might be beneficial for injury prevention. However, further research is needed to support this claim.

\section{PSYCHOLOGICAL DISTRESS AND MALADAPTIVE COPING IN OLYMPIC-LEVEL SWIMMERS FOLLOWING POSTPONEMENT OF THE 2020 OLYMPIC GAMES DUE TO COVID-19}

${ }^{1}$ Nicholas Hooper, ${ }^{1}$ Curtis Reiber, ${ }^{1}$ Seth Cheatham, ${ }^{2}$ Tessa Johnson. 'Virginia Commonwealth University, Richmond, USA; ${ }^{2}$ Moss Rehabilitation Institute, Philadelphia, USA

\subsection{6/bjsports-2021-IOC.136}

Background Elite-level athletes are vulnerable to psychological distress due to rigorous training and competitive schedules. Psychological screening is a preventative strategy to target athletes at risk for maladaptive coping and psychopathology; however, psychological screening is rarely implemented in elite sport environments.

Objective To assess psychological distress (i.e., anxiety, psychological strain, and burnout) and coping behavior in Olympiclevel swimmers following a major athletic stressor: postponement of the 2020 Olympics due to COVID-19.

Design A retrospective mixed-methods design was used.

Setting An online psychological assessment and a follow-up telehealth semi-structured interview were completed.

Participants Participants $(n=14)$ included international swimmers who: achieved a top 10 time; placed in the top 3 in their 2019 national meet; or qualified for the World Championships.

Assessment of Risk Factors Anxiety, psychological strain, and burnout were evaluated as risk factors for maladaptive coping. Outcome Measurements Assessment included Generalized Anxiety Disorder-7 (GAD-7), Athlete Psychological Strain Questionnaire (APSQ), and Athletic Burnout Questionnaire (ABQ) scales. Coping behaviors were derived from interview content (available for 12 of the 14 participants).

Results Findings (mean \pm SD) indicated mild anxiety (6.29 \pm $4.87)$ and severe psychological strain $(22.71 \pm 4.83)$. Higher psychological strain associated with higher emotional and physical exhaustion - a subscale of the ABQ ( $r=0.791 ; \mathrm{p}=$ $0.001)$. A subset of athletes $(n=5)$ reported moderate-to-severe anxiety. Most athletes $(\mathrm{n}=9)$ practiced maladaptive coping (75\%) (e.g., behavioral and mental disengagement); however, maladaptive coping was more frequent in athletes with greater psychological distress.

Conclusions Several athletes reported levels of anxiety and psychological strain that, according to APSQ and GAD-7 guidelines, necessitated clinical evaluation. Higher psychological distress coincided with more frequent maladaptive coping. Findings urge implementation of psychological screening in elite athletics to better support athletes' mental health and prevent development of maladaptive coping and psychopathology.

\section{ABSTRACT WITHDRAWN}

148 PERCEIVED INJURY RISK AMONG ELITE TRACK \& FIELD ATHLETES — A QUESTIONNAIRE-BASED STUDY

Maria Mereman. The Swedish School of Sport and Health Sciences, Stockholm, Sweden

10.1136/bjsports-2021-IOC.137 